\title{
Realidad Aumentada como herramienta de mejora de la inteligencia espacial en estudiantes de educación secundaria
}

\section{Augmented Reality as a tool for improving spatial intelligence in secondary education students}

\author{
Francisco del Cerro Velázquez \\ Universidad de Murcia. Murcia. España \\ fcerro@um.es \\ Ginés Morales Méndez \\ Universidad de Murcia. Murcia. España \\ gines.morales@um.es
}

\section{RESUMEN}

En el presente estudio se muestra una experiencia de innovación educativa llevada a cabo con alumnado de bajo rendimiento de tercer curso de Educación Secundaria Obligatoria, en la materia de Tecnologías. Centrando el eje del estudio entorno a una unidad didáctica vinculada a la expresión gráfica, con el fin de mejorar su grado de habilidad espacial. En el desarrollo de esta experiencia ha sido seleccionado como muestra de estudio el grupo de tercer curso, que presenta a nivel general mayor dificultad para asimilar este tipo de conocimientos.

Se describe de manera detallada como se ha innovado trabajando contenidos vinculados a la expresión gráfica: las perspectivas de los objetos; a través de herramientas de realidad aumentada. Para ello, se ha recurrido a una estrategia innovadora de enseñanza-aprendizaje centrada en el empleo de herramientas TIC, entre las que las que destaca la utilización de teléfonos inteligentes por parte del alumnado.

Presentamos la descripción detallada de la experiencia, así como los principales resultados observados tras su realización. Entendemos que inciden en la necesidad de introducir la realidad aumentada en el proceso de enseñanza-aprendizaje, como una estrategia educativa innovadora apoyada en el uso de los smartphones.

Palabras clave: Realidad aumentada, aprendizaje autónomo, inteligencia espacial, tecnologías, Educación Secundaria Obligatoria.

\footnotetext{
ABSTRACT:

This study presents an experience of educational innovation conducted with students with low achievements in their third school year of Secondary Education, in the subject of Technology. Focusing the center of the study in the teaching unit linked to the graphic expression, in order to improve their degree of space ability. In the development of this experience, we selected as a study sample, the group of third course, which presents on global level greater issues to assimilate this type of knowledge.

It describes in a detailed way, how has been innovated working contents linked to graphic expression: object perspectives; through augmented reality tools. For that, an innovative teachinglearning strategy has been used, focused on the use of ICT tools, including the use of smartphones by students.

We present a detailed description of the experience, as well as the main results observed after its realization. We understand that influences the need to introduce augmented reality into the teaching-learning process, as an innovative strategy supported by the use of smartphones.
} 
Keywords: Augmented Reality, autonomous learning, space intelligence, technologies, Secondary Education.

\section{Introducción}

A pesar de que los libros de texto y apuntes aún representan un papel dominante en la enseñanza, actualmente se están empleando nuevas herramientas TIC. Éstas actúan como facilitadoras de la docencia de los profesores y del aprendizaje para los estudiantes. El empleo del ordenador, como soporte físico, es una realidad a la que sumamos en estos últimos años, el uso de las tablets e, incluso, de los smartphones. Estamos ante un contexto, en el ámbito educativo, que favorece e invita a la inclusión de las TIC en los procesos de enseñanza-aprendizaje (e-a). Además, son cada vez más los docentes comprometidos que incorporan en su día a día dichos soportes en el aula.

En este sentido hay que señalar que la comunicación multidireccional es una de las características del mundo actual. La sociedad ha sufrido cambios que la han convertido en una red de comunicaciones, donde tomar y dar información es algo cada vez más sencillo. Por ello, la oferta de la interactividad se nos ofrece como algo muy apetecible y ha sido acogida de buen grado por parte de los usuarios (Ruiz Davis y Polo Serrano, 2012). Quinn (2000), citado por Fombona Cadavieco et al (2012), destacando la gestión informática portátil como el primer paso para la interactividad, conectividad total y el proceso electrónico en red de datos e imágenes simultáneas a la experiencia real. Facer et al (2004) y Willians et al (2005), a través de sus experiencias, resaltan como el uso de recursos móviles para el aprendizaje modifica el ambiente de enseñanza al convertir cualquier escenario en un entorno innovador y colaborativo.

Además las tecnologías actuales, como los dispositivos móviles, los entornos virtuales o la realidad aumentada (en adelante RA) presentan un potencial para descubrir y proporcionar nueva información en cualquier ámbito (Carmigniani et al, 2010). Añaden, Holzinger, Nischelwitzer y Meisenberger (2005), que una tendencia cada vez más frecuente en educación, como es el aprendizaje deslocalizado, que soluciona el problema actual de espacio-tiempo en el aprendizaje, no resuelto por el eLearning, lo solucionan el uso de dispositivos que permitan movilidad; como es el caso de la telefonía móvil. Ésta ofrece la posibilidad de construir esquemas flexibles en educación, tanto en tiempo como en espacio. Estamos ante un modelo de aprendizaje electrónico móvil o m-Learning, término acuñado por primera vez por Quinn (2000).

No obstante, las actividades diseñadas con estos soportes, deben sustentarse en estrategias educativas específicas, para que sean efectivas y se relacionen con las habilidades cognitivas que desarrollen los estudiantes (Ramos, Herrera y Ramírez, 2010).

Podemos destacar que una de las metas principales propuestas por los académicos de Horizon Project, que participan y ayuda a la regulación de la educación en la Unión Europea, es la inclusión para los próximos años de los trabajos en el dominio de la RA en centros educativos tanto superiores como de Educación Secundaria y Bachillerato (Johnson, Brown y Adams, 2014).

La situación actual se presenta, sin ninguna duda, como un escenario muy interesante para el desarrollo de aplicaciones educativas móviles, que difícilmente sería factible Realidad Aumentada como herramienta de mejora de la inteligencia espacial en estudiantes de educación secundaria. Francisco del Cerro Velázquez y Ginés Morales Méndez. 
realizar, tan solo hace unos años (Fiolhais y Trinidad, 2003; Cardoso, 2010). Por ello, hemos de aprovecharlas, de este modo daremos respuesta a una sociedad que nos demanda una educación diversa, que ofrezca soluciones educativas de calidad y en igualdad de oportunidades al alumno con necesidades específicas de apoyo (Martínez, del Cerro y Morales, 2014). En este sentido, y para este tipo de estudiantes, se ofrece una estrategia para afrontar unos contenidos específicos del currículo de la materia de Tecnologías.

Para ello, en este trabajo vamos a presentar una experiencia con alumnado de Educación Secundaria Obligatoria que ofrece ciertas dificultades en determinado tipo de contenidos vinculados con la inteligencia espacial, aprovechando el potencial que los dispositivos móviles representan como soporte para permitir utilizar estrategias de e-a basadas en RA. Posiblemente, uno de los campos con más futuro y más posibilidades en cualquier área o entorno educativo. Se trata de una tecnología emergente, que ofrece un amplio abanico en retos, especialmente en la educación y donde las prácticas educativas utilizando estas tecnologías todavía no afianzadas, están proporcionando resultados positivos, generando una mayor implicación del alumnado en los procesos de e-a.

\section{Códigos QR y Realidad Aumentada}

Los códigos QR (Quick Response) surgidos en la empresa Denso Wave, subsidiaria de Toyota en 1994, son definidos por Gamboa (2012) como una imagen, generalmente una matriz de puntos, en la que se almacena y activa información de respuesta rápida. La información codificada en estos códigos puede ser cualquier tipo de dato multimedia. En nuestra experiencia los utilizaremos como modulo para almacenar y activar los modelos 3D en RA.

Podemos definir brevemente la tecnología de RA como una técnica que consiste en añadir información virtual sobre un escenario real. Una definición más completa de este término consiste en señalar que la RA es un proceso que se realiza en tiempo real en función de lo que captura una cámara de un dispositivo, y se establece, además, una relación espacial entre la información virtual y su entorno real. Los elementos esenciales necesarios para poder visualizar contenido en RA consisten en un ordenador o un dispositivo móvil, una cámara y una aplicación que traduzca los datos de RA. Aclarar, que si la información que se va a añadir al entorno real está ubicada en la red se precisará de conexión a Internet.

Entendemos, que cuando hablamos de información adicional o virtual añadida sobre entornos reales, nos referimos a cualquier tipo de información susceptible de ser digitalizada, como es el caso de los textos, imágenes, vídeos, audios, enlaces web o imágenes en 3D, como hemos indicado anteriormente.

Algunos autores defienden que los códigos QR son un tipo de RA, denominándolos incluso RA en 2 dimensiones, utilizando como argumento que también enlazan con contenidos digitales. Otros señalan que no se pueden mezclar ambos conceptos, ya que la información que ofrecen los códigos QR no se ubica en entornos reales (Estebanell et al, 2012). En cuanto a los requisitos que debe cumplir un sistema de RA, Azuma (1997) señala que debe combinar a elementos reales y virtuales, ser interactivo en tiempo real y registrarse en $3 \mathrm{D}$.

Realidad Aumentada como herramienta de mejora de la inteligencia espacial en estudiantes de educación secundaria. Francisco del Cerro Velázquez y Ginés Morales Méndez. 


\section{Razones para incidir en el estudio y mejora de la inteligencia espacial en los estudiantes de educación secundaria}

En la etapa de educación secundaria, la mayoría de psicólogos, investigadores educativos y profesores centran su atención en analizar y mejorar las habilidades de lectura, comprensión y escritura, además de las disciplinas relacionadas con las materias de matemáticas y ciencias. Pero, ¿debería dedicarse parte de este tiempo en mejorar la habilidad espacial del alumnado? Son varios los motivos clave para que así sea.

El primero de ellos es que la inteligencia espacial se considera una inteligencia clave dentro de las conocidas como inteligencias múltiples (Gardner, 1983), desempeñando un papel único en el aprendizaje de nuevos conocimientos, mejorando de esta forma la capacidad mental para generar, girar y transformar imágenes visuales (Kell, Lubinski, Benbow y Steinger, 2013).

En segundo lugar, de acuerdo con las investigaciones basadas en este tipo de inteligencia en relación con los procesos cognitivos, se concreta que la habilidad espacial es una parte fundamental en el desarrollo del pensamiento verbal. Son varios los ejemplos extraídos de múltiples publicaciones que demuestran de forma analítica que la visualización espacial es una habilidad clave para una correcta evolución de las capacidades cognitivas y de la inteligencia general en niños y adolescentes (Carroll, 1993). De acuerdo con los resultados obtenidos en diversas pruebas de diagnóstico, se demuestra una alta dependencia de la inteligencia espacial para la comprensión de contenidos espacial/matemático en niños en edad escolar (Bornstein, 2009), así como en chimpancés (Herrmann, Hernández-Lloreda, Call, Hare y Tomasello, 2010).

En tercer lugar, el pensamiento espacial ayuda a razonar en campos que, aparentemente, no están vinculados con la inteligencia espacial. Por ejemplo, a la hora de realizar, entender o relacionar mapas mentales, esquemas, gráficos o croquis; utilizamos de forma indirecta habilidades basadas en la inteligencia lógica-matemática y espacial, siendo numerosas las técnicas de estudio que utilizan esta metodología de e-a. Estas herramientas de aprendizaje son consideradas de gran valor por su referencia a la construcción de conocimientos, desarrollo de pensamiento, así como para fomentar otros valores educativos (Ontoria, 2006).

Por último, la capacidad espacial juega un papel fundamental dentro de los programas educativos vinculados a ciencia, tecnología, ingeniería y matemáticas, materias conocidos como CTIM o STEM, cuyas metodologías educativas han impulsado la integración de la tecnología de RA en la educación (Schelly et al., 2015). El progreso y rendimiento en disciplinas CTIM está fuertemente ligado con la mejora de la capacidad para razonar sobre hipótesis espaciales y su comportamiento (Shea, Lubinski y Benbow, 2001). Además, los niños y adolescentes que tienen una inteligencia espacial elevada tienden a tener un mayor interés y motivación por los proyectos CTIM desarrollados en el aula y en su gran mayoría eligen realizar estudios superiores relacionados con estas disciplinas (Wai, Lubinski y Benbow, 2009).

Realidad Aumentada como herramienta de mejora de la inteligencia espacial en estudiantes de educación secundaria. Francisco del Cerro Velázquez y Ginés Morales Méndez. 


\section{Objetivos del estudio}

A través de esta experiencia se pretende diseñar una estrategia de e-a innovadora, a la vez que efectiva, a través de la tecnología de RA, donde los estudiantes con una habilidad espacial baja y rendimiento académico inferior deben adquirir y afianzar su propia autonomía. Pasando el alumnado de esta forma, a ser el verdadero protagonista en el proceso de su aprendizaje con un determinado tipo de contenidos vinculados con la inteligencia espacial, que tradicionalmente ha venido ofreciéndoles una cierta dificultad en cuanto a su asimilación. Por lo tanto, este estudio se centra en analizar y comparar los beneficios sobre la capacidad espacial del alumnado con bajo rendimiento a través del uso de material didáctico en RA frente a las aportaciones de los materiales didácticos bidimensionales de uso tradicional. Por último, se evaluarán los resultados de los posibles cambios en el alumnado a través de un modelo de estudio cuantitativo pretest-postest, adaptado para esta experiencia educativa y teniendo en cuenta dos de los indicadores de la inteligencia espacial: la rotación y la visualización espacial.

\section{Metodología empleada en el estudio}

\subsection{Sujetos participantes}

La experiencia se ha llevado a cabo con un grupo de 23 alumnos de $3^{\circ}$ curso de Educación Secundaria Obligatoria en el marco de la materia de Tecnologías, siendo 13 chicos y 10 chicas. Esta materia se imparte durante 3 sesiones semanales de 55 minutos cada una.

El grupo seleccionado $\left(3^{\circ} \mathrm{C}\right)$ corresponde al único de los 3 grupos de $3^{\circ} \mathrm{ESO}$ existentes en el centro educativo, que no tiene estudiantes integrados en la enseñanza bilingüe. Destacamos que los conocimientos académicos previos vinculados a la habilidad espacial de dicho alumnado, en los contenidos que van a ser trabajados, son claramente más bajos con respecto a los de los otros dos grupos (gráfico 1), como quedó de manifiesto en el cuestionario pretest que se llevó a cabo al inicio de la unidad didáctica vinculada con los contenidos de expresión gráfica.

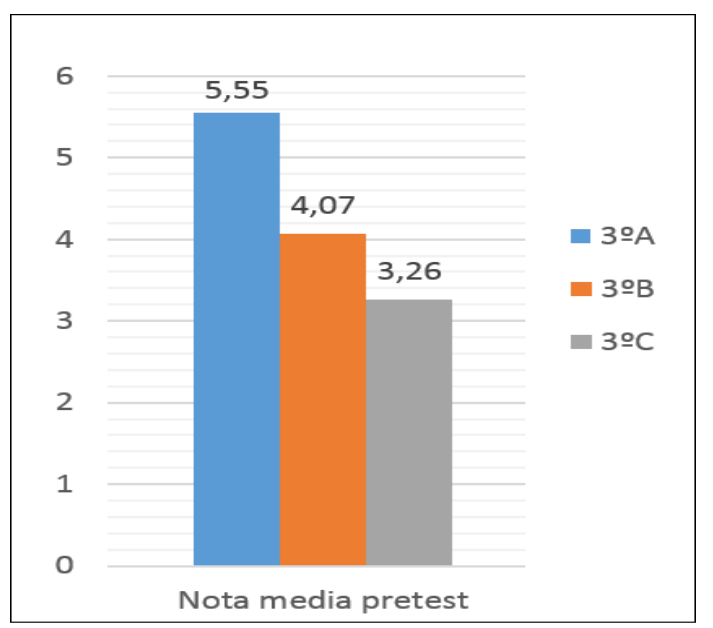

Gráfico 1. Resultados medios del cuestionario pretest por clase vinculados a la inteligencia espacial previa de los grupos de $3^{\circ} \mathrm{ESO}$

Los alumnos y alumnas se dividieron en grupos de 2 (excepto un grupo de 3) y realizaron el trabajo principalmente en clase (figura 1). Sus edades se encuentran

Realidad Aumentada como herramienta de mejora de la inteligencia espacial en estudiantes de educación secundaria. Francisco del Cerro Velázquez y Ginés Morales Méndez. 
comprendidas entre los 13 y los 17 años. La edad más repetida (moda) es 14 años y la media se corresponde con 14,29 años.

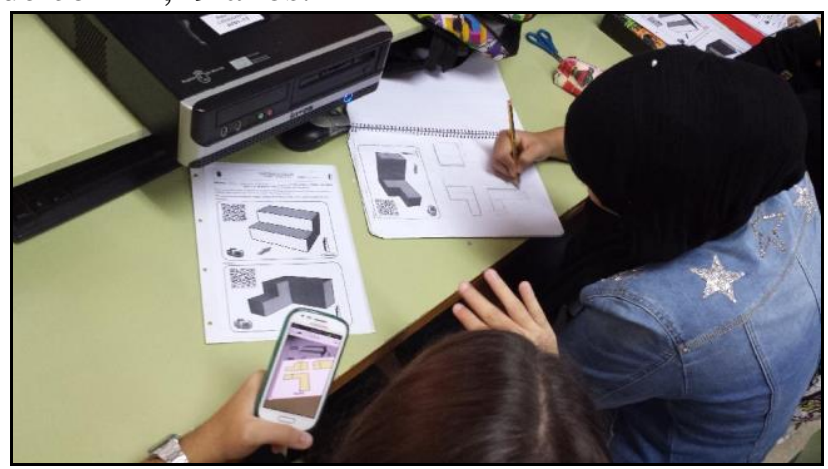

Figura 1: Pareja de alumnas durante un momento de la experiencia

\subsection{Recursos utilizados}

La herramienta fundamental para el desarrollo de esta experiencia con RA es el smartphone, que es uno de los 3 tipos de soportes de la RA, según Fombona Cadavieco (2012), junto con el ordenador tradicional y los equipos específicos de RA. Se trata de dispositivos portátiles miniaturizados que actúan como pequeñas mini computadoras e incorporan cámaras de captura o escaneo de imágenes. Pudiendo reproducir el efecto de RA y presentar en la pantalla del dispositivo el resultado. Por tanto, antes de iniciar la experiencia, se nos presentan varias situaciones a tener en cuenta y que deben ser afrontadas:

Por un lado, nos encontramos con la prohibición en el centro educativo, del uso de teléfonos móviles. Situación, por otro lado, bastante generalizada en los centros educativos del entorno y que ha sido provocada por el mal uso dado, en algunos casos aislados, por parte de estudiantes. Esta situación provocó la necesidad de realizar una solicitud especial para la autorización por parte de la dirección del centro al alumnado inmerso en la experiencia, para que pudiera traer de casa y disponer de su smartphone en el centro educativo durante el desarrollo de la experiencia. Únicamente durante los 3 días de la semana que tenían clase de Tecnologías y, exclusivamente, durante dicho periodo lectivo. Probablemente, sea esta necesidad uno de los principales hándicaps institucionales que se presentan cuando la estrategia metodológica innovadora precisa de soportes aún no muy aceptados o consolidados por el docente en general, como es el caso del uso del teléfono móvil como recurso didáctico. No obstante, los smartphones que debían traer al centro el alumnado tenían que disponer de unos requisitos mínimos: poseer datos móviles y capacidad de descarga de una aplicación app gratuita decodificadora de RA (Junaio, 2017), disponible tanto para Android como para iOS.

Los gráficos 2, 3 y 4 reflejan la disponibilidad de smartphones por el alumnado y sus familias. Nos encontramos que el 86,9\% disponía del mismo; el 84,6\% de los chicos, 11 teléfonos, y el 90,0\% de las chicas, 9 teléfonos. Pero, realmente, y a efectos de poder ser utilizados durante la experiencia, solamente se disponía de 13 dispositivos con conexión de datos móviles: 5 eran de chicos y 8 de chicas (gráfico 4). Esto último condicionaría el tipo de agrupamiento en el alumnado participante, estando distribuidos durante la experiencia por parejas.

Realidad Aumentada como herramienta de mejora de la inteligencia espacial en estudiantes de educación secundaria. Francisco del Cerro Velázquez y Ginés Morales Méndez. 

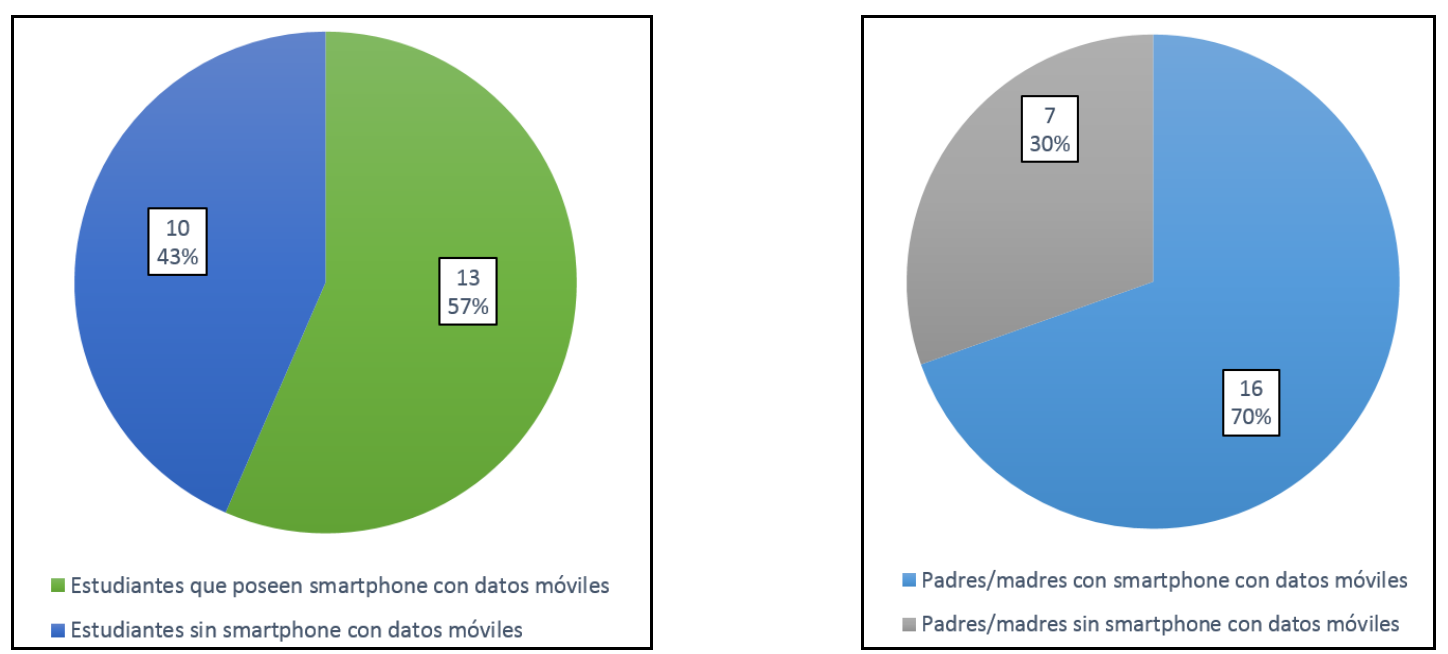

Gráfico 2 (izqda.): Alumnado con o sin smartphone

Gráfico 3 (dcha.): Número de familias con o sin smartphone

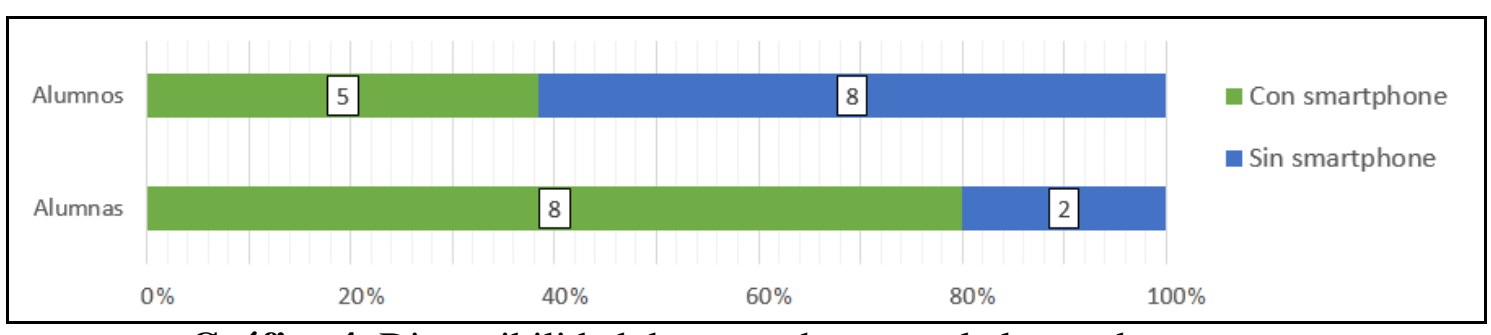

Gráfico 4: Disponibilidad de smartphone en el alumnado por sexo

\subsection{Desarrollo técnico}

La secuenciación técnica seguida para poder recrear las figuras 3D en RA, se corresponde con la presentada en el siguiente esquema (figura 2):

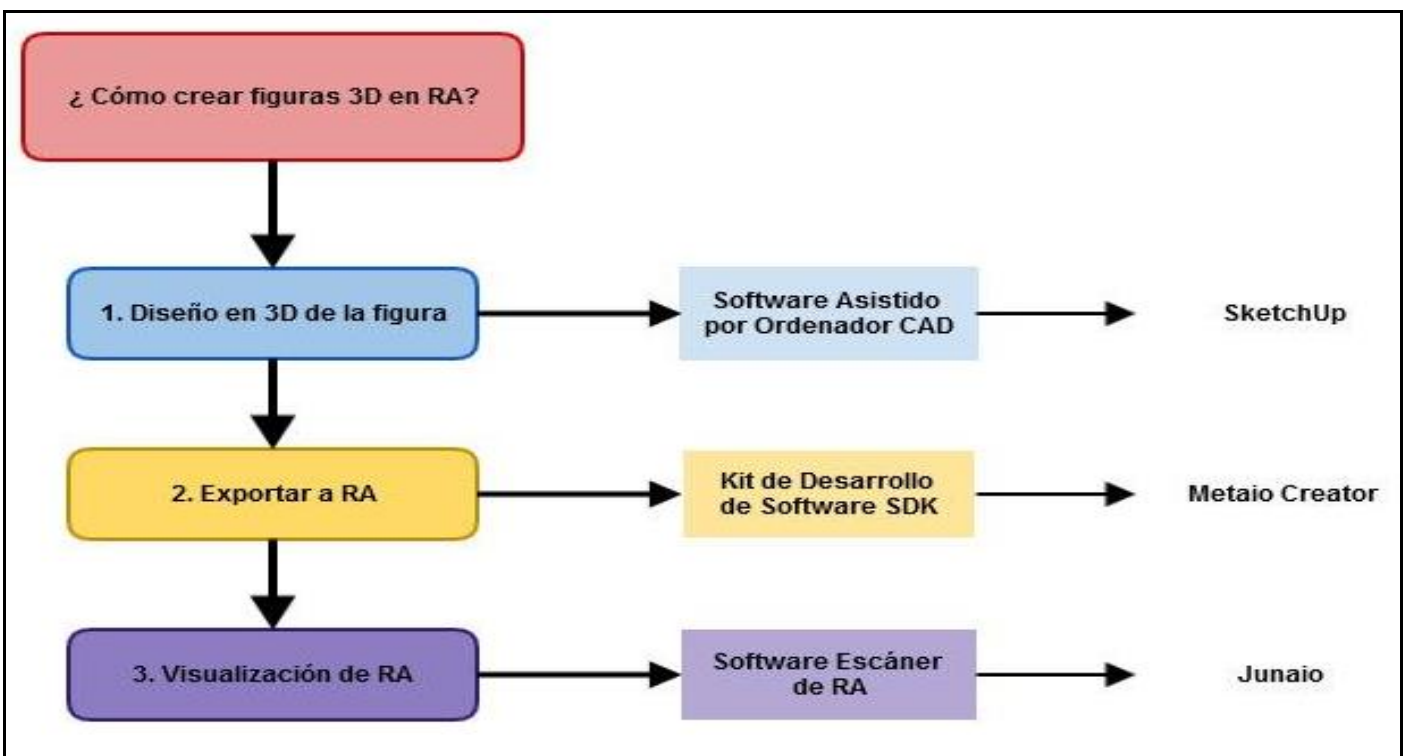

Figura 2: Secuenciación para la creación de figuras 3D en RA

En primer lugar, hemos de proceder a la digitalización del objeto para realizar la construcción de un modelo tridimensional empleando un software CAD 3D. Utilizamos la técnica de reconstrucción por imágenes de superficie, empleándose imágenes Realidad Aumentada como herramienta de mejora de la inteligencia espacial en estudiantes de educación secundaria. Francisco del Cerro Velázquez y Ginés Morales Méndez. 
tomadas del objeto desde diferentes ángulos para reunir la información exterior del mismo. En esta experiencia se ha optado por diseñar/digitalizar las piezas mediante el software asistido por ordenador (SketchUp Maker, 2017). Los factores que han hecho decantarnos por este programa son su interfaz sencilla, los bajos requisitos técnicos para su uso y su disponibilidad (versión gratuita). Las caras de las piezas fueron coloreadas con la intención de que alumnado asociase sus colores con cada una de sus vistas (figura 3).

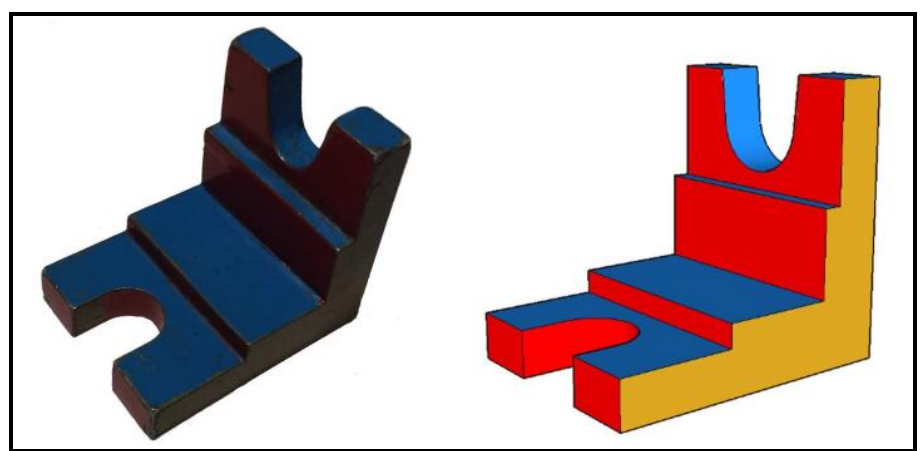

Figura 3: La única pieza física existente en el aula sin digitalizar (izqda). Pieza ya digitalizada (dcha)

Una vez diseñadas las figuras, las debemos guardar como modelo en 3D. Exportamos a un modelo 3D, adquiriendo un formato .obj.

A continuación hemos de vincular el modelo que hemos creado a una imagen, marca o código y convertirlo en contenido de RA. Para efectuarlo, hacemos uso de un kit de desarrollo de software de RA (Metaio Creator, 2017), que nos proporciona herramientas avanzadas para recrear entornos en RA de una forma sencilla, permitiendo de manera gratuita (en su versión básica) asociar fácilmente contenidos digitales a marcadores.

Para realizar este proceso, abrimos el programa Metaio Creator, cliqueamos en el botón "Trackables" situado en la parte inferior de su interfaz, y seguidamente en "Image Tracking". A continuación escogemos la imagen, código o marca que vamos a asociar a nuestro modelo en 3D (figura 4). En nuestro caso hemos aprovechado para incorporar el código QR que nos proporcionará más adelante el programa a la marca-imagen.

Una vez cargada la imagen, cliqueamos en el icono "3D model" situado dentro de la barra de herramientas "Resources", donde se nos deberá abrir una ventana de selección en la que debemos escoger el archivo ".obj" que contiene el modelo 3D. Una vez cargado el diseño, tan solo queda por ajustar el tamaño y la posición del objeto 3D a la marca (figura 5), y cliquear en "Create" para subir el trabajo realizado a la nube "Metaio Cloud". 


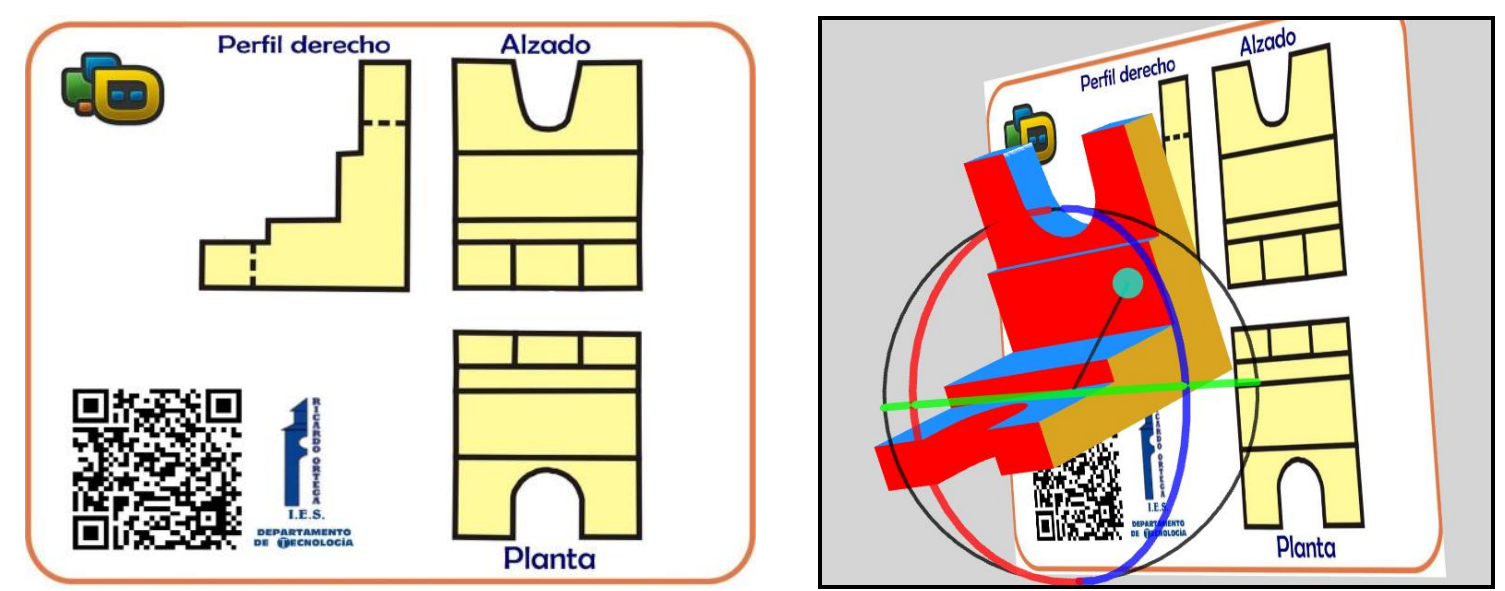

Figura 4 (izqda.): Marca asignada a una figura

Figura 5 (dcha.): Asociación del modelo 3D a una marca

Finalmente el programa nos proporcionará un código QR que debemos escanear con la aplicación gratuita para dispositivos Android e iOS Junaio. Una vez capturado este código con el dispositivo móvil, los estudiantes enfocarán con las cámaras de sus dispositivos a la marca que hemos asignado al modelo y de esta forma podrán interactuar con el modelo a la vez que visualizarlo libremente en RA desde todas sus perspectivas.

\subsection{Dinámica establecida}

Los grupos reciben una hoja de ejercicios al inicio de cada sesión, una hoja o ficha de ejercicios, construida con los recursos TIC comentados anteriormente. Cada una con dos propuestas de trabajo (dos piezas definidas por sus vistas), que se encuentran graduadas por su dificultad. Las fichas más básicas obedecen a piezas sencillas, donde todas sus vistas principales son planas, visibles y con proyección ortogonal. Paulatinamente se van introduciendo piezas más complejas que incorporan planos inclinados, curvos y, finalmente, vistas ocultas y piezas que presentan una mayor dificultad por su complejidad.

El trabajo es realizado por parejas de estudiantes. Situación condicionada por la disponibilidad únicamente de 14 smartphones con datos móviles (51,87\% del grupo). Se han empleado 8 sesiones, utilizándose únicamente el smartphone desde la $2^{\mathrm{a}}$ hasta la $7^{\mathrm{a}}$. La $1^{\text {a }}$ sesión se dedicó a trabajar aspectos introductorios a los contenidos que iban a ser tratados con RA y a la información sobre el método de trabajo a seguir durante las siguientes sesiones, cumplimentar el cuestionario inicial e indicarles las instrucciones para descargar la app Junaio. La $8^{a}$ sesión fue empleada para atender a cuestiones finales y evaluar la experiencia a través de un cuestionario de evaluación final.

\section{Resultados y análisis}

Para el desarrollo de la experiencia con RA se han elaborado unos materiales integrados con un soporte TIC de gran interés, permitiendo el uso individualizado de los contenidos. Se han generado de esta forma un total de 10 fichas de trabajo, conteniendo cada una de ellas 2 ejercicios prácticos de obtención de vistas de figuras a través de tecnología RA, y habiendo sido distribuidas al alumnado en formato papel A4 (figura 6). Por tanto, éstos materiales son capaces de generar un importante grado de autonomía

Realidad Aumentada como herramienta de mejora de la inteligencia espacial en estudiantes de educación secundaria. Francisco del Cerro Velázquez y Ginés Morales Méndez. 
en el alumnado (figura 7), siempre que se disponga como soporte de un teléfono móvil inteligente o, también, una tableta. Se hallan graduados en cuanto a su dificultad, de modo que permiten que cada estudiante-usuario pueda afrontar de un modo más cercano y seguro sus propios retos. Además, se produce una multiplicidad de materiales, siendo considerados como "materiales aumentados" (figura 8), que físicamente no se hallarían disponibles en un aula por su coste económico y que, sin embargo, ahora se encuentran disponibles, no solo en el aula, sino también fuera de ella.

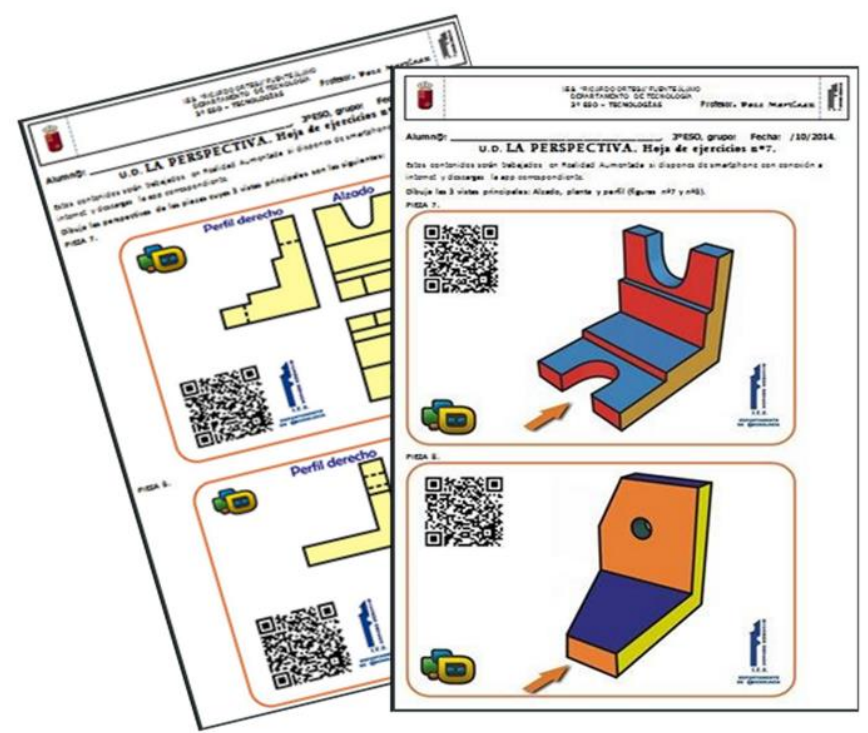

Figura 6: Dos de las fichas de trabajo elaboradas para el alumnado
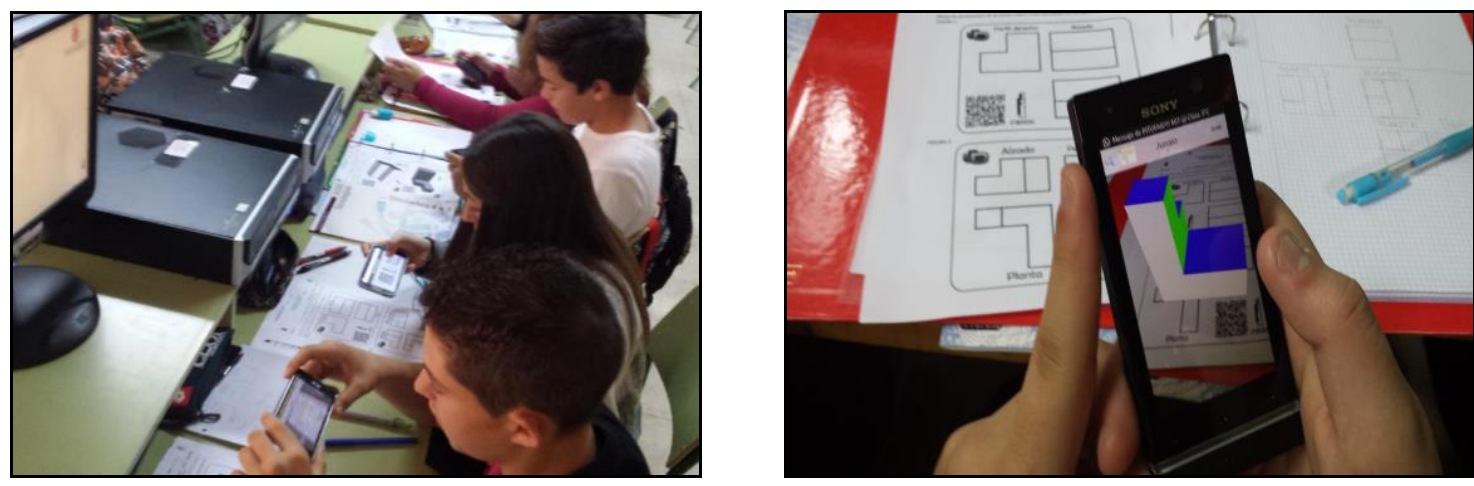

Figura 7 (izqda.): Estudiantes trabajando en el aula

Figura 8 (dcha.): Objeto visto en RA en el smartphone del estudiante

Los datos recogidos en las pruebas pretest y postest se analizaron y compararon utilizando el software estadístico Statistical Package for the Social Sciences (SPSS, 2017) mediante la realización de una prueba tipo t-student o test-t para averiguar la existencia de diferencias significativas. De esta forma se evalúa el impacto del empleo de actividades en RA para la mejora de la capacidad de visualización y rotación espacial del alumnado. Todo ello ha culminado con una calificación media en el análisis comparativo de los cuestionarios pretest-postest para evaluar el impacto del empleo de herramientas de RA para la mejora de la capacidad de visualización y rotación espacial del alumnado de 6,12 puntos (tabla 1). Resultados muy favorables, teniendo en cuenta el punto de partida de la muestra de 3,26 puntos.

Realidad Aumentada como herramienta de mejora de la inteligencia espacial en estudiantes de educación secundaria. Francisco del Cerro Velázquez y Ginés Morales Méndez. 


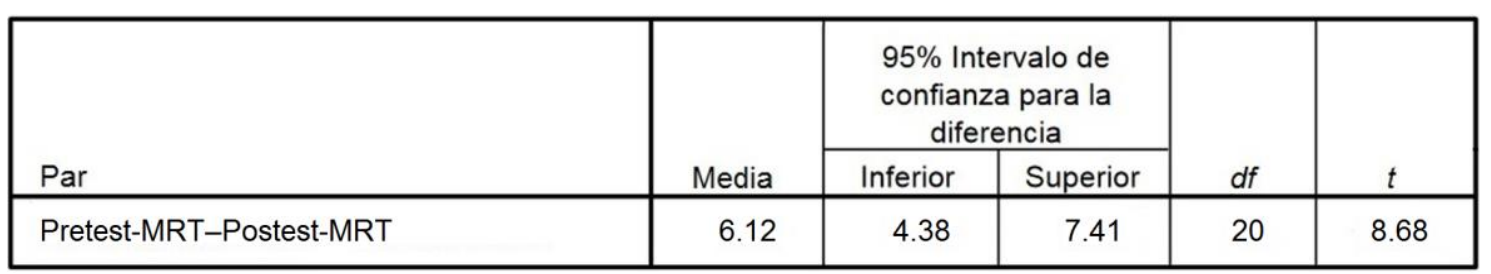

Tabla 1: T-test Pretest-Postest en los cuestionarios de inteligencia espacial

En cuanto a las respuestas obtenidas en el cuestionario de satisfacción, el alumnado manifiesta mayoritariamente, en un $82,60 \%$, estar muy satisfecho con la experiencia llevada a cabo (gráfico 5). Siendo muy alto el número de estudiantes que considera que la experiencia con RA le ha ayudado de modo considerable a aprender mejor este tipo de contenidos $(78,26 \%)$. También es muy elevado el número de estudiantes a los que les gustaría repetir la experiencia empleando RA en otros contenidos de la materia de Tecnologías.

En general, manifiestan haber trabajado con una gran motivación e interés, tanto en clase como en casa. El 69,56\% de las familias de la muestra disponen de smartphone con datos móviles lo que también ha facilitado y/o asegurado el uso de dichos dispositivos previa descarga de la app Junaio.

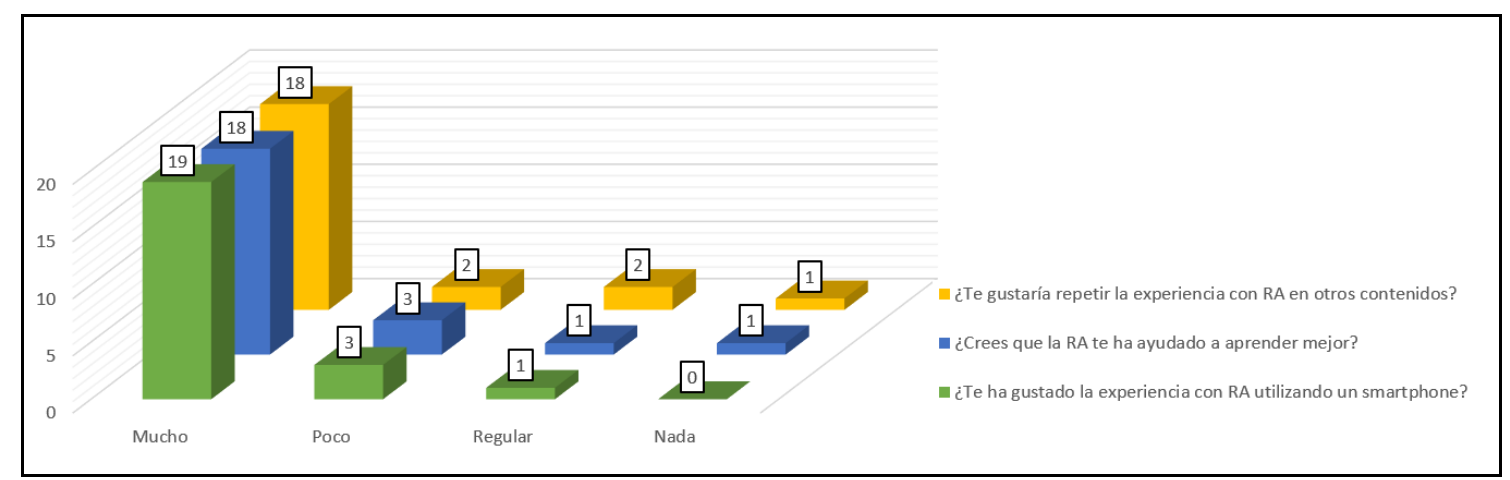

Gráfico 5: Resultados del cuestionario de satisfacción

\section{Discusión y conclusiones}

Atendiendo a los resultados obtenidos en esta experiencia con RA, centrada en trabajar con un determinado tipo de contenidos, que tradicionalmente han ofrecido cierta dificultad al alumnado en ESO, especialmente aquel con bajo rendimiento en actividades altamente vinculadas con la inteligencia espacial, destacamos:

Por un lado, la RA es una herramienta o recurso TIC emergente e innovador, que mejora el aprendizaje de los contenidos y, en concreto de los contenidos específicos de Tecnologías de ESO, cuestión ésta que resulta muy interesante para alumnado que presenta cierto grado de resistencia a la asimilación de los mismos. En este sentido, tomando como referencia la diferencia significativa entre las puntuaciones obtenidas en las pruebas de pretest y el postest, los resultados nos indican que la serie de actividades desarrolladas utilizando tecnología de RA son útiles para la mejora de la habilidad de rotación y visualización espacial en alumnado que parte con una inteligencia espacial baja. A pesar de ello, su uso aún no está muy extendido en las aulas, lo que permite entender que nos encontramos ante una estrategia educativa con una gran potencialidad

Realidad Aumentada como herramienta de mejora de la inteligencia espacial en estudiantes de educación secundaria. Francisco del Cerro Velázquez y Ginés Morales Méndez. 
para mejorar el proceso de e-a en los próximos años y en cualquier materia y, fundamentalmente, en todas las materias donde la inteligencia espacial es especialmente interesante, como son las materias relacionadas con la Tecnología, la Ingeniería, la Arquitectura, el Diseño, la Expresión Gráfica, el Arte, la Geografía, etc.

Por otro lado, la implementación de una aplicación didáctica, basada en recursos TIC, para el alumnado de $3^{\circ}$ de la ESO en la materia Tecnologías ha sido una experiencia enriquecedora y motivadora, que nos ha permitido comprobar la actitud positiva y tremendamente receptiva del alumnado ante este tipo de experiencias. Es evidente que esta motivación viene provocada por el hecho de estar utilizando dispositivos móviles con los que las nuevas generaciones están actualmente muy familiarizados, además de su satisfacción al comprobar que a medida que se avanzaba en la experiencia iban comprendiendo y asimilando los contenidos con facilidad. En este sentido, insistimos que llama especialmente la atención la respuesta de la totalidad de los estudiantes y su alto grado de implicación, así como, incluso, la complicidad de las familias.

Igualmente, con el uso de la RA se fomenta en el alumnado la capacidad de indagación e investigación, a la vez que se potencia el desarrollo del trabajo autónomo del estudiante en su apremdizaje. El alumnado afronta cada uno de los retos que se le van presentando con la seguridad de que la RA le facilitará alcanzarlos.

Finalmente, el grado de satisfacción de este alumnado con bajo rendimiento general, junto a los resultados globales obtenidos y su interés por repetir la experiencia con otros contenidos diferentes nos corroboran la idea de haber correspondido certeramente a los intereses y expectativas del mismo.

Presentación del artículo: 30 de abril de 2017

Fecha de aprobación: 15 de junio de 2017

Fecha de publicación: 30 de junio de 2017

Del-Cerro-Velázquez, F. y Morales-Méndez, G. (2017). Realidad Aumentada como herramienta de mejora de la inteligencia espacial en estudiantes de educación secundaria. RED. Revista de Educación a Distancia, 53. Consultado el (dd/mm/aaaa) en http://www.um.es/ead/red/54

\section{Financiación}

Esta investigación no ha recibido ninguna subvención específica de los organismos de financiación en los sectores públicos, comerciales o sin fines de lucro.

Realidad Aumentada como herramienta de mejora de la inteligencia espacial en estudiantes de educación secundaria. Francisco del Cerro Velázquez y Ginés Morales Méndez. 


\section{Bibliografía}

Azuma, R. T. (1997). A survey of augmented reality. Presence 6 (4), pp. 355-385.

Bornstein, M.H. (2009). The mind of the preschool child: The intelligence-school interface. Handbook of early child development and early education: Research to practice, pp. 123-142. New York: Guilford.

Cardoso, A. V. (2010). Apredizagem científica e tecnologíca no ensino médio: una experiência de design de material didáctico em 3D. Anais do II Simpôsio Nacional de Ensino de Ciência e Tecnologia. Cuitiba, pp. 1-17.

Carmigniani, J., Dueht, B., Anisetti, M., Ceravolo,P., Daminani, E. \& Ivkovic, M. (2010). Augmented reality technologies, systems and applications. Multimedia Tools and Applications, 51 (1), pp. 341-377.

Carrol, J.B. (1993). Human cognitive abilities: A survey of factor analytic studies. New York: Cambridge University Press.

Del Cerro, F., Martínez, F. y Morales, G. (2014). La construcción virtual a través de Build with Chrome frente a la real, como estrategia para la adaptación curricular en alumnus con necesidades educativas especeiales. En J. Navarro et al. (cords). III Congreso Nacional de Buenas Prácticas en Atención a la Diversidad. Murcia, España.

Estebanell, M.; Ferrés, J.; Cornellà, P. y Codina, D. (2012). Realidad aumentada y códigos QR en educación. En Tendencias emergentes en educación con TIC, pp. 277-320. Barcelona: Editorial Espiral.

Facer, K., Joiner, R., Stanton, D., Reid, J., Hull, R. \& Kirk, D. (2004). Savannah: Mobile gaming and learning? Journal of Computer Assisted Learning, 20 (6), pp. 399-409.

Fiolhais. C. y Trinidad, J. (2003). Física no Computador: o Computador como uma Ferramienta no Ensino e na Aprendizagem das Ciências Físicas. Revista Brasileira de Enisno de Física, 25 (3).

Fombona, J., Pascual, M., Madeira, A. y Madeira Ferreira, M. (2012). Realidad aumentada, una evolución de las aplicaciones de los dispositivos móviles. Pixel-Bit. Revista de Medios y Educación, (41), pp. 1978-210.

Gardber, H. (1983). Frames of mind: The theory of multiple intelligences. New York: Basic Books.

Gamboa, J. L. (2012). El uso de códigos QR en la enseñanza. En, Tendencias emergentes en la educación con TIC, pp. 401-425. Barcelona: Editorial Espiral.

Herrmann, E., Hernández-Lloreda, M. V., Call, J., Hare, B. \& Tomasello, M. (2010). The estructure of individual differences in congnitive abilities of children and chimpanzees. Psychological Science, 21, pp.102-110.

Holzinger, A., Nischelwitzer, A. \& Meisenberger, M. (2005). Lifelong-learning support by M-Learning: example scenarios. ACM eLearn Magazine, 5.

Realidad Aumentada como herramienta de mejora de la inteligencia espacial en estudiantes de educación secundaria. Francisco del Cerro Velázquez y Ginés Morales Méndez. 
Johnson, L., Brown, M. R \& Adams, S. (2014). The 2014 Horizon Report. The New Media Consortium. Austin, Texas. Disponible en: http://cdn.nmc.org/media/2014nmc-horizon-report-he-EN-SC.pdf. Fecha de consulta: 11 de octubre del 2014.

Junaio. (6.0.5.1). [Programa informático]. Munich, Alemania: Metaio GmbH.

Kell, H.J., Lubinski, D., Benbow, C.P. \& Steiger, J.H. (2013). Creativity and technical innovation: Spatial ability's unique role. Psychological science, 24(9), pp. 18311836.

Metaio Creator. (6.02). [Programa informático]. Munich, Alemania: Metaio GmbH.

Ontoria, A. (2006). Mapas Conceptuales. Una técnica para aprender. Madrid, Narcea.

Quinn, C. (2000). mLearning: Mobile Wireless, In-Your-Pocket Learning. Line zine. Learning in the new economy. Consultado el 14/12/2016 en: http://www.linezine.com/2.1/features/cqmmwiyp.htm

Ramos, A. I., Herrera, J. A. y Ramirez, M. S. (2010). Desarrollo de habilidades cognitivas cn aprendizaje móvil: un estudio de casos. Comunicar, 34, pp. 201-209.

Ruiz Davis, S. y Polo Serrano, D. (2012). La realidad aumentada como nuevo concepto de la publicidad online a través de los smartphones. Razón y Palabra (80).

Schelly, C., Anzolone, G., Wijnen, B., Pearce, J.M. (2015). Open-source 3-D printing technologies for education: bringing additive manufacturing to the classroom. $J$. Visual Lang. Comput. 28, pp. 226-237.

Shea D.L., Lubinski, D., \& Benbow, C.P. (2001). Importance of assessing spatial ability in intellectually talented Young adolescents: A 20-year longitudinal study. Journal of Educational Psichology, 93, pp.604-614.

SketchUp Maker. (16.1.1449). [Programa informático]. Sunnyvale, California: Trimble Navigation.

Statistical Package for the Social Sciences (SPSS). (24.0). [Programa informático]. Nueva York: International Business Machines (IBM).

Wai, J., Lubinski, D. \& Benbow, C.P. (2009). Spatial ability for STEM domains: Aligning over 50 years of comulative psychological knowledge solifies its importance. Journal of Educational Psychology, 101, pp. 817-835.

Willians, M., Jones, O., Fleuriot, C. \& Wood, L. (2005). Children and emerging wireless technologies: Investigating the potential for saptial practice. En ACM Conference on Human Factor in Computing Systems, pp. 819-828. Portland: ACM Press.

Realidad Aumentada como herramienta de mejora de la inteligencia espacial en estudiantes de educación secundaria. Francisco del Cerro Velázquez y Ginés Morales Méndez. 